\title{
A GENERALIZED PROBLEM IN WEIGHTED APPROXIMATION*
}

BY

\author{
DUNHAM JACKSON
}

1. Introduction. In a recent note, the writer has discussed the convergence of the trigonometric sums $T_{n}(x)$ determined by the condition that

$$
\int_{0}^{2 \pi} \varrho(x)\left[f(x)-T_{n}(x)\right]^{2} d x
$$

shall be a minimum, where $f(x)$ is a given continuous function, and $\varrho(x)$ is a given bounded function with a positive lower bound. The purpose of the present paper is to discuss the somewhat less simple problem of convergence that arises if the greatest lower bound of $\varrho(x)$ is zero. There will be occasion incidentally to develop certain other topics, which have some independent interest: in $\S \S 2$ and 3 , the theory of the existence and uniqueness of discontinuous approximating functions of a high degree of generality, and in $\S 5$, some corollaries of Bernstein's theorem on the derivative of a trigonometric sum.

2. General theorem on existence of an approximating function. Let

$$
p_{1}(x), p_{2}(x), \ldots, p_{n}(x)
$$

be $n$ functions of $x$, defined and linearly independent for $a \leqq x \leqq b$, but otherwise completely arbitrary. It is well known, and readily proved, $\ddagger$ that if

* Presented to the Society, October 27 and December 29, 1923.

$\dagger \mathrm{D}$. Jackson, Note on the convergence of weighted trigonometric series, Bulletin of the American Mathematical Society, vol. 29 (1923), pp. 259-263. This paper will be designated by the letter $\mathrm{A}$; the reader is referred to it for other bibliographical citations.

$\ddagger$ Suppose the determinant were identically zero. On this assumption, let $r+1$ (equal to or less than $n$ ) be a number such that the determinants similarly formed with $r+1$ of the $n$ functions and $r+1$ points are identically zero, for every choice of the $r+1$ functions, while it is possible to find $r$ of the functions and $r$ points for which the corresponding $r$-rowed determinant is not zero. Without loss of generality, the $r$ functions may be denoted by $p_{1}, \ldots, p_{r}$, and the points by $x_{1}, \ldots, x_{r}$. The $(r+1)$-rowed determinant having $p_{1}\left(x_{j}\right), p_{2}\left(x_{j}\right), \ldots, p_{r+1}\left(x_{j}\right)$ for its $j$ th row, $j=1,2, \ldots, r$, and $p_{1}(x), p_{2}(x), \ldots$. $\ldots, p_{r+1}(x)$ for its last row, is identically zero when $x_{1}, \ldots, x_{r}$ are held fast and $x$ is 
$x_{1}, \ldots, x_{n}$ are allowed to range independently over the interval, the determinant having $p_{k}\left(x_{j}\right)$ for its typical element is not identically zero. This being recognized, let $x_{1}, \ldots, x_{n}$ henceforth stand for a particular set of points for which the determinant is different from zero, the points being chosen once for all and then held fast. Let $D$ stand for the absolute value of the determinant formed with $x_{1}, \ldots, x_{n}$, so that $D>0$, and let $D_{1}$ be the greatest absolute value attained by any of its first minors.

Let

$$
\varphi(x)=c_{1} p_{1}(x)+c_{2} p_{2}(x)+\cdots+c_{n} p_{n}(x)
$$

be an arbitrary linear combination of the $p$ 's with constant coefficients. If $\boldsymbol{H}_{1}$ is the largest of the numbers $\left|\varphi\left(x_{1}\right)\right|, \ldots,\left|\varphi\left(x_{n}\right)\right|$, and if the $c$ 's are regarded as obtainable by Cramer's rule from the simultaneous equations

$$
c_{1} p_{1}\left(x_{j}\right)+c_{2} p_{2}\left(x_{j}\right)+\cdots+c_{n} p_{n}\left(x_{j}\right)=\varphi\left(x_{j}\right) \quad(j=1,2, \ldots, n),
$$

it is clear that $\left|c_{k}\right| \leqq n D_{1} H_{1} / D$ for $k=1,2, \ldots, n$. It is still not necessary to assume that the $p$ 's are bounded, and even if they are, $|\varphi(x)|$ may take on values larger than $H_{1}$ at other points than $x_{1}, \ldots, x_{n}$. For the applications in this paper, however, it will be sufficient to use the following form of

Sibirani's Lemma.* If the functions $p_{1}, \ldots, p_{n}$ are linearly independent and bounded for $a \leqq x \leqq b$, and if $H$ is an upper bound for $|\varphi(x)|$ in the interval, then

$$
\left|c_{k}\right| \leqq P H \quad(k=1,2, \ldots, n)
$$

where $P$ is a number independent of the coefficients $c_{k}$.

allowed to vary over the interval; its expansion according to the elements of the last row gives a linear relation connecting $p_{1}(x), \ldots, p_{r+1}(x)$, in which the coefficient of $p_{r+1}$ is not zero. This standard proof is repeated here just for the sake of assuring the reader that it does not require that the $p$ 's be continuous, bounded, measurable, or otherwise restricted in any way. The identical vanishing of the determinant is clearly a necessary as well as a sufficient condition for linear dependence, with the same complete generality.

* F. Sibirani, Sulla rappresentazione approssimata delle funzioni, An n a li di M a t e ma tica, ser. 3, vol. 16 (1909), pp. 203-221; see p. 208. A different proof, somewhat less general in its scope, has been given by the writer: D. Jackson, On functions of closest approximation, these Transactions, vol. 22 (1921), pp. 117-128; see pp. 118-119. The proof given here is Sibirani's own, loc. cit., though he was not concerned with so general a statement of the conclusion. Cf. also L. Tonelli, I polinomi d'approssimazione di Tchebychev, the same Annali, ser. 3, vol. 15 (1908), pp. 47-119; pp. 61-62. The method is applicable also, as Sibirani makes clear, to functions of more than one variable. 
A different number $P$ might be obtained by a different choice of the points $x_{1}, \ldots, x_{n}$, but the essential thing is that such a number exists.

It will be assumed from now on that the functions $p_{k}$ are bounded and measurable, and that they satisfy something more than the bare requirement of linear independence, to the extent that, for every choice of the coefficients $c_{k}$ (other than $\left.c_{k}=0, k=1,2, \ldots, n\right)$, there is a point set of positive measure throughout which $\varphi(x)$ is different from zero. For brevity, unless a better term is already in use, they may then be called properly independent.

Let $H$ be the least upper bound of $|\varphi(x)|$. The functions $p_{k}$ being given, $H$ is then a function of the coefficients $c_{k}$. It will be shown that $H$ is a continuous function of the $c$ 's. For the time being, the dependence of $\varphi$ on the $c$ 's will be indicated explicitly by the notation $\varphi\left(x, c_{1}, \ldots, c_{n}\right)$. Let $Q$ be a number such that $\left|p_{k}(x)\right| \leqq Q$ for $a \leqq x \leqq b$ and for $k=1,2, \ldots, n$. If $c$ is the largest of the numbers $\left|c_{k}\right|$,

$$
\left|\varphi\left(x, c_{1}, \ldots, c_{n}\right)\right| \leqq n c Q .
$$

Let $\varepsilon$ be an arbitrary positive quantity. Let two sets of coefficients be considered, $\left(c_{k}\right)$ and $\left(c_{k}+\Delta c_{k}\right)$, and suppose that $\left|\Delta c_{k}\right| \leqq \varepsilon /(2 n Q)$ for $k=1,2, \ldots, n$. Then, since $\varphi$ depends linearly on the $c$ 's,

$\left|\varphi\left(x, c_{1}+\Delta c_{1}, \ldots, c_{n}+\Delta c_{n}\right)-\varphi\left(x, c_{1}, \ldots, c_{n}\right)\right|=\left|\varphi\left(x, \Delta c_{1}, \ldots, \Delta c_{n}\right)\right|<\frac{\varepsilon}{2}$

throughout $(a, b)$. As $\left|\varphi\left(x, c_{1}, \ldots, c_{n}\right)\right| \leqq H\left(c_{1}, \ldots, c_{n}\right)$ throughout the interval, it follows that

$$
\left|\varphi\left(x, c_{1}+\Delta c_{1}, \ldots, c_{n}+\Delta c_{n}\right)\right|<H\left(c_{1}, \ldots, c_{n}\right)+\frac{\varepsilon}{2}
$$

that is,

$$
H\left(c_{1}+\Delta c_{1}, \ldots, c_{n}+\Delta c_{n}\right)<H\left(c_{1}, \ldots, c_{n}\right)+\frac{\varepsilon}{2} .
$$

On the other hand, since $H$ is the least upper bound of $\varphi$, there will be a point $x_{0}$ where

$$
\left|\varphi\left(x_{0}, c_{1}, \ldots, c_{n}\right)\right|>H\left(c_{1}, \ldots, c_{n}\right)-\frac{\varepsilon}{2} .
$$

Then $\left|\varphi\left(x_{0}, c_{1}+\Delta c_{1}, \ldots, c_{n}+\Delta c_{n}\right)\right|>H\left(c_{1}, \ldots, c_{n}\right)-\varepsilon$, and

$$
H\left(c_{1}+\Delta c_{1}, \ldots, c_{n}+\Delta c_{n}\right)>H\left(c_{1}, \ldots, c_{n}\right)-\varepsilon .
$$

This proves the continuity of $H$ as a function of the $c$ 's. 
It will be shown next that if $J$ denotes the integral

$$
J=\int_{a}^{b}|\varphi(x)|^{m} d x
$$

for a fixed $m>0$, and if the coefficients in $\varphi$ are allowed to vary in such a way that $J$ remains bounded, the coefficients themselves will remain bounded also. Sibirani's lemma establishes the fact that the sets of coefficients for which the least upper bound $H$ has a given fixed value, $H=1$, say, correspond to a bounded point set in the space of $n$ dimensions having $c_{1}, \ldots, c_{n}$ for coördinates. It follows from the last paragraph that this point set is closed. On the other hand, $J$ itself is a continuous function of the coefficients. Among the functions $\varphi$ for which $H=1$, then, there will be at least one for which the value of $J$ is a minimum. Let this minimum be $\operatorname{denoted}$ by $A$. It is certain that $A>0$; the coefficients in $\varphi$ can not all be zero when $H=1$, and therefore, since the $p$ 's are assumed properly independent, $\varphi$ is different from zero throughout a set of positive measure. If $\varphi$ is an arbitrary linear combination of the $p$ 's (not identically zero), and $H$ the least upper bound of its absolute value, the least upper bound of $|\varphi / H|$ is 1 , and

$$
\int_{a}^{b}\left|\frac{\varphi(x)}{H}\right|^{m} d x \geqq A, \quad J=\int_{a}^{b}|\varphi(x)|^{m} d x \geqq A H^{m} .
$$

Let $c$ once more be equal to the largest $\left|c_{k}\right|$; then

$$
c \leqq P H, \quad H \geqq \frac{c}{P}, \quad J \geqq \frac{A c^{m}}{P^{m}}, \quad c \leqq P\left(\frac{J}{A}\right)^{1 / m},
$$

or, if $P_{1}$ stands for the number $P / A^{1 / m}$, independent of the $c$ 's,

$$
c \leqq P_{1} J^{1 / m}
$$

This proves that the $c$ 's are bounded when $J$ is bounded.

Let $f(x)$ be an arbitrary function, bounded and measurable in $(a, b)$. It is possible now to consider the minimizing of the integral

$$
J^{\prime}=\int_{a}^{b}|f(x)-\varphi(x)|^{m} d x
$$


This integral is a continuous function of the coefficients in $\varphi$. Suppose first that $f$ and the $p$ 's constitute a set of $n+1$ properly independent functions. If $J_{0}^{\prime}$ is a particular value taken on by $J^{\prime}$, the coefficients for which $J^{\prime} \leqq J_{0}^{\prime}$ correspond to a point set which is bounded, by the preceding paragraph, and closed, because of the continuity of $J^{\prime}$. There is consequently a determination of the coefficients for which $J^{\prime}$ is a minimum. If $f$ and the $p$ 's are not properly independent, a linear relation connecting them must involve $f$ with a non-vanishing coefficient, because of the independence of the $p$ 's by them selves. This means that $f$ is identically equal to a linear combination of the $p$ 's in $(a, b)$, except possibly for a point set of measure zero. If this linear combination of the $p$ 's is taken for $\varphi, J^{\prime}$ is reduced to zero, which is then clearly its minimum value. In summary:

If the functions $p_{1}(x), \ldots, p_{n}(x)$ are bounded, measurable, and properly independent for $a \leqq x \leqq b$, if $f(x)$ is bounded and measurable in the same interval, and if $m$ is a positive constant, there is at least one determination of the coefficients in the linear combination $s=c_{1} p_{1}+\cdots+c_{n} p_{n}$ for which the value of the integral

is a minimum.*

$$
\int_{a}^{b}|f(x)-\varphi(x)|^{m} d x
$$

A function $\varphi$ which gives the integral its minimum value will be called an approximating function.

3. General theorem on uniqueness of the approximating function, $m>1$. A familiar type of argument will justify the assertion

If $m>1$ in the hypothesis of the preceding theorem, the approximating function is uniquely determined.

Suppose, if possible, that $\varphi_{1}$ and $\varphi_{2}$ are two approximating functions which are not identically equal. Let the dependence of $J^{\prime}$ on $\varphi$ be indicated by the notation $J^{\prime}(\varphi)$, and let the minimum value of $J^{\prime}$ be denoted by $\gamma$. Then

$$
J^{\prime}\left(\varphi_{1}\right)=J^{\prime}\left(\varphi_{2}\right)=\gamma
$$

\footnotetext{
* This theorem would still be true if the $p$ 's were not independent. The truth of the statement is trivial if they are all identically zero. Otherwise, there is a properly independent subset among them, on which the remaining functions of the set are linearly dependent, except possibly for point sets of measure zero. There is an approximating function in terms of the properly independent subset, by the theorem as proved above, and this is at the same time an approximating function in terms of the original set of $p$ 's. But the hypothesis of independence is necessary for the theorem of uniqueness in the next section.
} 
Let $\varphi_{3}=\frac{1}{2}\left(\varphi_{1}+\varphi_{2}\right)$, so that $f-\varphi_{3}=\frac{1}{2}\left[\left(f-\varphi_{1}\right)+\left(f-\varphi_{2}\right)\right]$. In consequence of the property which is described geometrically by saying that the graph of the function $Y=|X|^{m}$ is everywhere concave upwards (when $m>1$ ),

$$
\left|f-\varphi_{9}\right|^{m} \leqq \frac{1}{2}\left[\left|f-\varphi_{1}\right|^{m}+\left|f-\varphi_{2}\right|^{m}\right]
$$

for all values of $x$, and the relation is an actual inequality at every point where $\varphi_{1} \neq \varphi_{2}$. If $\varphi_{1}$ and $\varphi_{2}$ are not identical, they differ in value over a point set of positive measure, by the hypothesis of proper independence of the $p$ 's, and the sign of inequality holds in (1) at all points of this set. Consequently

$$
J\left(\varphi_{3}\right)<\frac{1}{2}\left[J\left(\varphi_{1}\right)+J\left(\varphi_{2}\right)\right] ;
$$

that is, $J\left(\varphi_{3}\right)<\gamma$, which is impossible.

4. Existence and uniqueness of solution of a problem in weighted trigonometric approximation. The general theorems above have been derived here primarily for the sake of a more specific application. Let $\varrho(x)$ be a function which is bounded and measurable, and of period $2 \pi$; let it be assumed that $\varrho(x) \geqq 0$ everywhere, and that $\varrho(x)>0$ over a point set of positive measure in an interval of length $2 \pi$. Let $f(x)$ be a function which is bounded and measurable, and of period $2 \pi$, but otherwise unrestricted, for the present. Let $T_{n}(x)$ be a trigonometric sum of the $n$th order, ${ }^{*}$ and let $m$ be a positive constant. The discussion will be concerned now with the integral

$$
\int_{0}^{2 \pi} \rho(x)\left|f(x)-T_{n}(x)\right|^{m} d x .
$$

The most interesting case, mentioned in the introduction, is that in which $m=2$, but the method applies equally well to the more general problem.

The integral (2) can be written in the form

$$
\int_{0}^{2 \pi}\left|\sqrt[m]{\varrho(x)} f(x)-\sqrt[m]{\varrho(x)} T_{n}(x)\right|^{m} d x .
$$

\footnotetext{
* This will be understood throughout to mean a trigonometric sum of order $n$ at most.
} 
The function $\sqrt{\varrho(x)} f(x)$ is bounded and measurable for $0 \leqq x \leqq 2 \pi$. The expression $\bar{V} \overline{\varrho(x)} T_{n}(x)$ is a linear combination of the functions

$$
\bar{V} \overline{\varrho(x)}, \quad \sqrt[m]{\varrho(x)} \cos k x, \quad \sqrt[m]{\varrho(x)} \sin k x \quad(k=1,2, \ldots, n),
$$

and these functions are bounded, measurable, and properly independent for $0 \leqq x \leqq 2 \pi$, since, if $T_{n}(x)$ is any trigonometric sum of the $n$th order with coefficients not all zero, $\bar{V} \overline{\varrho(x)} T_{n}(x)$ is different from zero at all points of the set where $\rho \neq 0$, with the exception of $2 n$ points at most in any interval of length $2 \pi$. So the preceding theorems are applicable, with $n$ replaced by $2 n+1$, the functions $p_{k}(x)$ by the functions (3), and $f(x)$ by $V^{m} \overline{\varrho(x)} f(x)$, while $a=0, b=2 \pi$. The conclusion is as follows:*

Under the hypotheses stated at the beginning of the section, there will be at least one, and, when $m>1$, just one determination of the coefficients in $T_{n}(x)$, for which the value of the integral (2) is a minimum.t

5. Corollaries of Bernstein's Theorem. The convergence proof in the paper A depended essentially on

BeRnstein's THEOREm. $\ddagger$ If $T_{n}(x)$ is a trigonometric sum of order $n$, and if $\left|T_{n}(x)\right| \leqq L$ for all values of $x$, then $\left|T_{n}^{\prime}(x)\right|$ can never exceed $n L$.

This theorem is not immediately adequate in the present circumstances, inasmuch as it requires that the condition on $\left|T_{n}(x)\right|$ be satisfied for all values of $x$, or, what amounts to the same thing, throughout an interval of length $2 \pi$, if the conclusion is to be available over even a part of a period; and there will be occasion now to reason from a similar hypothesis, the validity of which

* Mr. J. Shohat informs me that he had obtained an analogous result some years ago, in an unpublished paper, for the case of polynomial approximation. The present method is of course applicable to the polynomial case also.

† When $m<1$, the determination is not necessarily unique; cf. D. Jackson, Note on an ambiguous case of approximation, these Transactions, vol. 25 (1923), pp. 333-337. This paper will be cited by the letter B. It can be regarded as dealing with a special case under the present discussion, in which $\rho$ is identically 1 . In this special case, the determination is unique when $m=1$; cf. D. Jackson, Note on a class of polynomials of approximation, these Transactions, vol. 22 (1921), pp. 320-326. The question of uniqueness for $m=1$ and for general $\rho$ will not be treated here.

$\ddagger$ Cf., e. g., D. Jackson, The general theory of approximation by polynomials and trigonometric sums, Bulletin of the American Mathematical Society, vol. 27 (1921), pp. 415-431; see pp. 421-422. The proof given in the Bulletin was quoted there as due originally to de la Vallée Poussin; it has since come to my attention that it had been published still earlier by Marcel Riesz, Eine trigonometrische Interpolationsformel und einige Ungleichungen für Polynome, Jahresbericht der Deutschen MathematikerVereinigung, vol. 23 (1914), pp. 354-368; see pp. 360-361. 
is granted only over an interval of length less than $2 \pi$. The relations that are needed can be derived indirectly from Bernstein's theorem, and it is the purpose of this section to supply them.

Let $P_{n}(x)$ be a polynomial of the $n$th degree, and $L$ the maximum of $\left|P_{n}(x)\right|$ for $-1 \leqq x \leqq 1$. Let $x=\cos \theta$. Then $P_{n}(x)=T_{n}(\theta)$ is a trigonometric sum of the $n$th order in $\theta$ (involving only cosines), and, by application of the theorem just quoted,*

$$
\begin{gathered}
\left|\sqrt{1-x^{2}} P_{n}^{\prime}(x)\right|=\left|\sin \theta P_{n}^{\prime}(\cos \theta)\right|=\left|T_{n}^{\prime}(\theta)\right| \leqq n L, \\
\left|P_{n}^{\prime}(x)\right| \leqq \frac{n L}{\sqrt{1-x^{2}}}
\end{gathered}
$$

for $-1<x<1$.

More generally, let $P_{n}(x)$ be a polynomial of the $n$th degree, and let $L$ be the maximum of $\left|P_{n}(x)\right|$ over an arbitrary interval $a \leqq x \leqq b$. Let $y=(2 x-a-b) /(b-a)$. Then $P_{n}(x)=Q_{n}(y)$ is a polynomial of the $n$th degree in $y$, and $L$ is the maximum of $\left|Q_{n}(y)\right|$ for $-1 \leqq y \leqq 1$, so that $\left|Q_{n}^{\prime}(y)\right| \leqq n L / \sqrt{1-y^{2}}$ throughout the interior of this interval. But $Q_{n}^{\prime}(y)=\frac{1}{2}(b-a) P_{n}^{\prime}(x)$, the accent denoting differentiation with respect to the indicated argument in each case; and, on the other hand,

$$
1+y=\frac{2(x-a)}{b-a}, 1-y=\frac{2(b-x)}{b-a}, \frac{1}{\sqrt{1-y^{2}}}=\frac{b-a}{2 \sqrt{(b-x)(x-a)}} .
$$

Consequently

$$
\left|P_{n}^{\prime}(x)\right| \leqq \frac{n L}{\sqrt{(b-x)(x-a)}}
$$

for $a<x<b$.

Now let $P_{n-1}(x)$ be a polynomial of degree $n-1$, and let $L$ be the maximum of $\left|\sqrt{1-x^{2}} P_{n-1}(x)\right|$ for $-1 \leqq x \leqq 1$. Let $x=\cos \theta$. Then $P_{n-1}(x)$ is a cosine sum of order $n-1$ in $\theta$. The result of multiplying this sum by $\sin \theta$ is a trigonometric sum of order $n$ (involving sines only), which may be denoted by $T_{n}(\theta)$. In terms of the original variables,

* Cf. M. Riesz, loc. cit., pp. 359-360; D. Jackson, On the convergence of certain trigonometric and polynomial approximations (to be cited below by the letter $\mathrm{C}$ ), these Transactions, vol. 22 (1921), pp. 158-166, see p. 162; and, originally, S. Bernstein, Sur l'ordre de la meilleure approximation des fonctions continues par des polynomes de degré donné, Mémoire couronné, Brussels, 1912, pp. 6-11. My paper, written before I knew of the existence of that of Riesz, should have contained a reference to the latter in connection with pp. 162-163. 
$T_{n}(\theta)= \pm \sqrt{1-x^{2}} P_{n-1}(x)$, so that $\left|T_{n}(\theta)\right| \leqq L$, and $\left|T_{n}^{\prime}(\theta)\right| \leqq n L$, for all values of $\theta$. Inasmuch as

$$
\left|T_{n}^{\prime}(\theta)\right|=\left|\sin \theta \frac{d}{d x} T_{n}(\theta)\right|=\left|\sqrt{1-x^{2}} \frac{d}{d x}\left[\sqrt{1-x^{2}} P_{n-1}(x)\right]\right|,
$$

it is seen that*

$$
\left|\frac{d}{d x}\left[\sqrt{1-x^{2}} P_{n-1}(x)\right]\right| \leqq \frac{n L}{\sqrt{1-x^{2}}}
$$

for $-1<x<1$.

Let $P_{n-1}(x)$ be a polynomial of degree $n-1$ again, but let $L$ this time be the maximum of $\left|\sqrt{(b-x)(x-a)} P_{n-1}(x)\right|$ for $a \leqq x \leqq b$. If the change of variable of the second paragraph preceding is carried through, it is found that the various factors $\frac{1}{2}(b-a)$ cancel each other, and the conclusion is that

$$
\left|\frac{d}{d x}\left[\sqrt{(b-x)(x-a)} P_{n-1}(x)\right]\right| \leqq \frac{n L}{\sqrt{(b-x)(x-a)}}
$$

for $a<x<b$.

These lemmas with regard to polynomials have been set down here for the sake of their application to trigonometric sums. Let $U_{n}(\theta)$ be a trigonometric sum of the $n$th order involving only cosines, and let $L$ be the maximum of $\left|U_{n}(\theta)\right|$ for $-\alpha \leqq \theta \leqq \alpha$, where $0<\alpha<\pi$. As $U_{n}(\theta)$ is a polynomial of the $n$th degree in $\cos \theta$, and $L$ the maximum of its absolute value for $\cos \alpha \leqq \cos \theta \leqq 1$, one of the preceding results is applicable, to the effect that

$$
\begin{aligned}
&\left|\frac{d}{d \cos \theta} U_{n}(\theta)\right| \leqq \frac{n L}{\sqrt{(1-\cos \theta)(\cos \theta-\cos \alpha)}} \\
&\left|U_{n}^{\prime}(\theta)\right| \leqq \frac{n L|\sin \theta|}{\sqrt{(1-\cos \theta)(\cos \theta-\cos \alpha)}} \\
&=n L \sqrt{\frac{1+\cos \theta}{\cos \theta-\cos \alpha}} \leqq \frac{\sqrt{2} n L}{\sqrt{\cos \theta-\cos \alpha}}
\end{aligned}
$$

The reasoning is valid in the first instance for $\cos \alpha<\cos \theta<1$; but as $U_{n}^{\prime}(\theta)$ and the last member on the right are continuous for $\theta=0$, the relation between them remains in force at this point also, and so holds throughout the interval $-\alpha<\theta<\alpha$.

* Cf. Bernstein, op. cit., pp. 17-19. 
The corresponding argument for a sum of sines appears to be materially less simple. Let $\nabla_{n}(\theta)$ be a trigonometric sum of the $n$th order involving only sines, and let $L$ be the maximum of $\left|V_{n}(\theta)\right|$ for $-\alpha \leqq \theta \leqq \alpha$, where $0<\alpha<\pi$, as before. The quotient $V_{n}(\theta) / \sin \theta$ can be expressed as a polynomial of degree $n-1$ in $\cos \theta$, and may be represented by $P_{n-1}(\cos \theta)$. Let $\alpha_{1}$ and $\alpha_{2}$ be two numbers between 0 and $\alpha$, so that $0<\alpha_{2}<\alpha_{1}<\alpha$, and so that $\alpha_{2}<\pi-\alpha$. For $\cos \alpha \leqq \cos \theta \leqq \cos \alpha_{2}$,

$$
\left|P_{n-1}(\cos \theta)\right|=\left|\frac{\nabla_{n}(\theta)}{\sin \theta}\right| \leqq \frac{L}{\sin \alpha_{2}}
$$

and, throughout the interior of the interval,

$$
\begin{aligned}
\left|\frac{d}{d \theta} P_{n-1}(\cos \theta)\right|=\left|\sin \theta P_{n-1}^{\prime}(\cos \theta)\right| & \leqq\left|P_{n-1}^{\prime}(\cos \theta)\right| \\
& \leqq \frac{(n-1) L}{\sin \alpha_{2} \sqrt{\left(\cos \alpha_{2}-\cos \theta\right)(\cos \theta-\cos \alpha)}}
\end{aligned}
$$

If $\theta$ is further restricted so that $\cos \alpha<\cos \theta \leqq \cos \alpha_{1}$, then $\cos \alpha_{2}-\cos \theta$ $\geqq \cos \alpha_{2}-\cos \alpha_{1}$, and it can be stated briefly that

$$
\left|\frac{d}{d \theta} P_{n-1}(\cos \theta)\right| \leqq \frac{\check{k}_{1} n L}{\sqrt{\cos \theta-\cos \alpha}}
$$

where $k_{1}$ is a number independent of $n$, and depending only on $\alpha_{1}$ and $\alpha_{2}$. In the same interval,

$$
\begin{gathered}
\left|V_{n}^{\prime}(\theta)\right|=\left|\frac{d}{d \theta}\left[\sin \theta P_{n-1}(\cos \theta)\right]\right| \\
=\left|\sin \theta \frac{d}{d \theta} P_{n-1}(\cos \theta)+\cos \theta P_{n-1}(\cos \theta)\right| \leqq \frac{k_{1} n L}{\sqrt{\cos \theta-\cos \alpha}}+\frac{L}{\sin \alpha_{2}} .
\end{gathered}
$$

If the second term in the last member is merged with the first term by means of the trivial relations

$$
1 \leqq n, \frac{1}{\sin \alpha_{2}}=\frac{\sqrt{\cos \theta-\cos \alpha}}{\sin \alpha_{2}} \cdot \frac{1}{\sqrt{\cos \theta-\cos \alpha}} \leqq \frac{\sqrt{2}}{\sin \alpha_{2}} \cdot \frac{1}{\sqrt{\cos \theta-\cos \alpha}}
$$


it is seen that

$$
\left|V_{n}^{\prime}(\theta)\right| \leqq \frac{k_{2} n L}{\sqrt{\cos \theta-\cos \alpha}}
$$

for $\cos \alpha<\cos \theta \leqq \cos \alpha_{1}$, the number $k_{2}$ depending once more on $\alpha_{1}$ and $\alpha_{2}$ and on nothing else.

For $\cos \alpha \leqq \cos \theta \leqq 1$,

$$
\left|\sin \theta P_{n-1}(\cos \theta)\right| \leqq L .
$$

But in this interval

$$
\begin{aligned}
|\sin \theta|=\sqrt{1-\cos ^{2} \theta} & =\sqrt{(1-\cos \theta)(\cos \theta-(-1))} \\
& >\sqrt{(1-\cos \theta)(\cos \theta-\cos \alpha)},
\end{aligned}
$$

and therefore

$$
\left|\sqrt{(1-\cos \theta)(\cos \theta-\cos \alpha)} P_{n-1}(\cos \theta)\right| \leqq L
$$

Let the quantity between the bars in the last relation be denoted by $W(\theta)$. By the last of the lemmas for polynomials,

$$
\left|\frac{d}{d \cos \theta} W(\theta)\right| \leqq \frac{n L}{\sqrt{(1-\cos \theta)(\cos \theta-\cos \alpha)}}
$$

for $\cos \alpha<\cos \theta<1$, whence

$$
\left|W^{\prime}(\theta)\right| \leqq \frac{n L|\sin \theta|}{\sqrt{(1-\cos \theta)(\cos \theta-\cos \alpha)}} \leqq \frac{\sqrt{2} n L}{\sqrt{\cos \theta-\cos \alpha}}
$$

It will be remembered that

$$
\nabla_{n}(\theta)=\sin \theta P_{n-1}(\cos \theta)= \pm \sqrt{\frac{1+\cos \theta}{\cos \theta-\cos \alpha}} W(\theta)
$$

The radical, which may be denoted by $Z(\theta)$, becomes infinite for $\theta=\alpha$. If $\theta$ is restricted so that $\cos \alpha_{1} \leqq \cos \theta<1$, however, both $Z(\theta)$ and its derivative will be bounded, and it can be asserted that 
$\left|V_{n}^{\prime}(\theta)\right|=\left|Z(\theta) W^{\prime}(\theta)+Z^{\prime}(\theta) W(\theta)\right|$

$$
\leqq \frac{k_{3} n L}{\sqrt{\cos \theta-\cos \alpha}}+k_{4} L \leqq \frac{k_{5} n L}{\sqrt{\cos \theta-\cos \alpha}},
$$

the numbers $k_{3}, k_{4}$, and $k_{5}$ depending only on $\alpha_{1}$. As far as the interval now under discussion is concerned, the denominator could of course be omitted, with suitable modification of the constants; it is retained for convenience in passing on to the main conclusion. The relation between the first and last members holds by continuity for $\theta=0$ as well.

Let $k_{6}$ be the larger of the numbers $k_{2}$ and $k_{5}$. As $\alpha_{1}$ and $\alpha_{2}$ can be chosen once for all when $\alpha$ is given, it may be considered that the $k$ 's, and in particular $k_{6}$, depend only on $\alpha$. The two preceding paragraphs, taken together, show that

$$
\left|V_{n}^{\prime}(\theta)\right| \leqq \frac{k_{6} n L}{\sqrt{\cos \theta-\cos \alpha}}
$$

for $-\alpha<\theta<\alpha$.

Finally, let $T_{n}(\theta)$ be an arbitrary trigonometric sum of order $n$. Let $L$ be the maximum of $\left|T_{n}(\theta)\right|$ for $-\alpha \leqq \theta \leqq \alpha, 0<\alpha<\pi$. Let

$$
U_{n}(\theta)=\frac{1}{2}\left[T_{n}(\theta)+T_{n}(-\theta)\right], \quad V_{n}(\theta)=\frac{1}{2}\left[T_{n}(\theta)-T_{n}(-\theta)\right]
$$

so that $U_{n}(\theta)$ is a sum of cosines, $V_{n}(\theta)$ a sum of sines, $T_{n}(\theta)=U_{n}(\theta)+V_{n}(\theta)$, and $\left|T_{n}^{\prime}(\theta)\right| \leqq\left|U_{n}^{\prime}(\theta)\right|+\left|\nabla_{n}^{\prime}(\theta)\right|$. It is seen that $\left|U_{n}(\theta)\right| \leqq L,\left|V_{n}(\theta)\right| \leqq L$, throughout the interval. The inequalities that have been.obtained for $\left|U_{n}^{\prime}(\theta)\right|$ and $\left|V_{n}^{\prime}(\theta)\right|$ separately can be combined; if the number $\sqrt{2}+k_{6}$, brought in by the combination, is denoted by $k_{0}$, the conclusion is that

$$
\left|T_{n}^{\prime}(\theta)\right| \leqq \frac{k_{0} n L}{\sqrt{\cos \theta-\cos \alpha}}
$$

for $-\alpha<\theta<\alpha$, the value of $k_{0}$ depending only on $\alpha$, not on $\theta$, or $n$, or the coefficients in $T_{n}(\theta)$.

The essence of this conclusion can also be expressed in a slightly different form, by the use of the relation

$$
\cos \theta-\cos \alpha=2 \sin \frac{\alpha-\theta}{2} \sin \frac{\alpha+\theta}{2} .
$$


Since $0<\alpha \pm \theta<2 \alpha<2 \pi$ throughout the interval in question,

$$
\frac{\sin \frac{1}{2}(\alpha \pm \theta)}{\frac{1}{2}(\alpha \pm \theta)}>\frac{\sin \alpha}{\alpha}>0
$$

and

$$
\cos \theta-\cos \alpha>\frac{\sin ^{2} \alpha}{2 \alpha^{2}}\left(\alpha^{2}-\theta^{2}\right)
$$

If $k_{0} \alpha \sqrt{2} / \sin \alpha$ is denoted by $k$, this means that

$$
\left|T_{n}^{\prime}(\theta)\right| \leqq \frac{k n L}{\sqrt{\alpha^{2}-\theta^{2}}}
$$

The last inequality can be adapted immediately, by a linear transformation, to an interval not symmetric with respect to the origin. With a slight change of notation, the result may be summarized in the following

LEмма. If $T_{n}(x)$ is a trigonometric sum of order $n$, and if $\left|T_{n}(x)\right| \leqq L$ for $\alpha \leqq x \leqq \beta$, where $0<\beta-\alpha<2 \pi$, then

$$
\left|T_{n}^{\prime}(x)\right| \leqq \frac{k n L}{\sqrt{(\beta-x)(x-\alpha)}}
$$

for $\alpha<x<\beta$, the multiplier $k$ depending only on the length of the interval, not on $x$, or $n$, or the coefficients in $T_{n}(x)$.

6. Convergence. With the notation and results of $\S 4$, and with the lemma of $\S 5$, some progress can be made in the study of the convergence of $T_{n}(x)$ toward $f(x)$ as $n$ becomes infinite. There will be occasion naturally to impose further restrictions on $f(x)$, which will be specified later. It will clearly be necessary also to introduce additional hypotheses either with regard to $\varrho(x)$ or with regard to the values of $x$ for which the convergence is to be established; for if $\rho(x)$ were to vanish identically throughout an interval, convergence to the value of $f(x)$ could not be proved at interior points of this interval, since $f(x)$ could be replaced by an altogether different function in the interval, without affecting the determination of $T_{n}(x)$, and the change could be made, in the interior of the interval at least, without violating the requirements of continuity* that are to be placed on $f(x)$. It will be assumed

* It will not be assumed anywhere in this paper that $f(x)$ is analytic. 
for convenience of expression that $m>1$, so that the approximating function is known to be uniquely determined, though the method is applicable in other cases also*.

As in other discussions of similar character, the problem can be simplified a little at the outset. Let $\varphi(x)$ be a function such that the difference between $f$ and $\varphi$ is identically equal to a trigonometric sum $t_{n}(x)$ of the $n$th order:

$$
\varphi(x)=f(x)-t_{n}(x)
$$

If $T_{n}(x)$ is for the moment an arbitrary trigonometric: sum of the $n$th order, and if

$$
\tau_{n}(x)=T_{n}(x)-t_{n}(x)
$$

the value of the integral (2) is the same as that of the corresponding integral with $f(x)$ and $T_{n}(x)$ replaced by $\varphi(x)$ and $\tau_{n}(x)$ respectively. In particular, if $T_{n}(x)$ is the approximating sum for $f(x)$-as will be understood henceforth-then $\tau_{n}(x)$ is at the same time the approximating sum for $\varphi(x)$. In other words, the approximating sums for $f(x)$ and $\varphi(x)$ are connected by the relation (5), and the errors $f(x)-T_{n}(x)$ and $\varphi(x)-\tau_{n}(x)$ are identical. It will be possible to choose $\varphi$ so that the latter error is more convenient to deal with directly.

By way of preliminary discussion, let $\varphi(x)$ be an arbitrary bounded and measurable function of period $2 \pi$, and $\tau_{n}(x)$ the approximating sum of the $n$th order for $\varphi(x), n \geqq 1$. Let $\varepsilon$ be the least upper bound of $|\varphi(x)|$. For the present, $\varepsilon$ may be large or small, although, as the notation suggests, a succession of functions $\varphi$ will ultimately be considered, with values of $\varepsilon$ approaching zero. Let it be supposed that there is an interval $\alpha \leqq x \leqq \beta$, throughout which (except possibly for a point set of measure zero) $\varrho(x) \geqq v>0$. Let $\rho(x) \leqq V$ for all valuest of $x$. Let $\mu$ be the maximum of $\left|\tau_{n}(x)\right|$ in the interval $\alpha \leqq x \leqq \beta$, and let $x_{0}$ be a point of this interval such that $\left|\tau_{n}\left(x_{0}\right)\right|=\mu$. It is to be shown that a relation of inequality connecting $\mu$ and $\varepsilon$ can be deduced without further specification of the function $\varphi$.

By the lemma of the preceding section,

$$
\left|\tau_{n}^{\prime}(x)\right| \leqq \frac{k n \mu}{\sqrt{(\beta-x)(x-\alpha)}}
$$

\footnotetext{
* Cf. the paper B, §4.

$\dagger$ Here again, exception may by made of a point set of measure zero, without affecting the values of the integrals involved, and so without affecting the essential conditions of the problem.
} 
for $\alpha<x<\beta$, the multiplier $k$ depending only on $\beta-\alpha$. Consequently, if $x$ is a point of the interval,

$$
\left|\tau_{n}(x)--\tau_{n}\left(x_{0}\right)\right| \leqq k n \mu\left|\int_{x_{0}}^{\alpha} \frac{d x}{\sqrt{(\beta-x)(x-\alpha)}}\right| .
$$

For definiteness, suppose that $\alpha \leqq x_{0} \leqq \frac{1}{2}(\alpha+\beta)$; the possibility that $x_{0}$ belongs to the other half of the interval is to be treated in essentially the same way. Let $h$ be a positive number $<\frac{1}{2}(\beta-\alpha)$. Then the interval $\left(x_{0}, x_{0}+h\right)$ is wholly contained in $(\alpha, \beta)$. For $x_{0} \leqq x \leqq x_{0}+h$,

$$
\left|\tau_{n}(x)-\tau_{n}\left(x_{0}\right)\right| \leqq k n \mu \int_{x_{0}}^{x_{0}+h} \frac{d x}{\sqrt{(\beta-x)(x-\alpha)}}
$$

The integrand is the reciprocal of the ordinate of a semicircle with its extremities on the $x$-axis at the points with abscissas $\alpha$ and $\beta$, so that the value of the integral from $x_{0}$ to $x_{0}+h$ is not greater than the value of the integral of the same integrand from $\alpha$ to $\alpha+h$; it would of course be easy, but perhaps superfluous, to give the details of an analytical proof. Furthermore, $\beta-x>\frac{1}{2}(\beta-\alpha)$ when $x$ is between $\alpha$ and $\alpha+h$, so that, in formulas,

$$
\begin{aligned}
& \int_{x_{0}}^{x_{0}+h} \frac{d x}{\sqrt{(\beta-x)(x-\alpha)}} \leqq \int_{\alpha}^{\alpha+h} \frac{d x}{\sqrt{(\beta-x)(x-\alpha)}} \\
& \leqq \sqrt{\frac{2}{\beta-\alpha}} \int_{\alpha}^{\alpha+h} \frac{d x}{\sqrt{x-\alpha}}=2 \sqrt{\frac{2 h}{\beta-\alpha}}, \\
&\left|\tau_{n}(x)-\tau_{n}\left(x_{0}\right)\right| \leqq k^{\prime} n \mu \sqrt{h},
\end{aligned}
$$

where $k^{\prime}$ is a new multiplier depending only on $\beta-\alpha$.

Now let* $h=1 /\left(4 k^{\prime 2} n^{2}\right)$. This $h$ will satisfy the requirement of being less than $\frac{1}{2}(\beta-\alpha)$, as soon as $n$ is sufficiently large; more precisely, this will be the case for $n \geqq n_{0}$, where $n_{0}$, like the $k$ 's, depends only on $\beta-\alpha, \operatorname{not}$ on $\rho$ or $\varphi$

* It is clear that $k^{\prime}>0$; for $k$, in the lemma of $\S 5$, is certainly not zero, and $k^{\prime}$ is equal to $k$ multiplied by a positive quantity. 
or anything else. As the ultimate question at issue is one of convergence for $n=\infty$, it may be assumed that $n \geqq n_{0}$ henceforth. Then, for $x_{0} \leqq x \leqq x_{0}+h$,

$$
\left|\boldsymbol{\tau}_{n}(x)-\boldsymbol{\tau}_{n}\left(x_{0}\right)\right| \leqq \frac{\mu}{2}, \quad\left|\tau_{n}(x)\right| \geqq \frac{\mu}{2} .
$$

This is on the hypothesis that $x_{0} \leqq \frac{1}{2}(\alpha+\beta)$; a similar argument applies if $x_{0}>\frac{1}{2}(\alpha+\beta)$, the conclusion holding for $x_{0}-h \leqq x \leqq x_{0}$, with the same values of $k^{\prime}, h$, and $n_{0}$ as before.

Let

$$
\gamma_{n}=\int_{0}^{2 \pi} \varrho(x)\left|\varphi(x)-\tau_{n}(x)\right|^{m} d x
$$

By the definition of $\varepsilon,|\varphi(x)| \leqq \varepsilon$ for all values of $x$. Let it be supposed for the moment that $\mu \geqq 4 \varepsilon$; the contrary hypothesis will be considered later. Then $|\varphi(x)| \leqq \mu / 4$, and the relations (7) imply that

$$
\left|\varphi(x)-\tau_{n}(x)\right| \geqq \frac{\mu}{4} .
$$

These relations hold at least throughout an interval of length $h$, where $h$ has the value specified above; the interval of length $h$ is contained in the interval $(\alpha, \beta)$, where $\varrho(x) \geqq v>0$, except possibly for a set of measure zero; and the integrand in the expression* for $\gamma_{n}$ is never negative. So it can be inferred that

$$
\gamma_{n} \geqq h v\left(\frac{\mu}{4}\right)^{m}=\frac{v \mu^{m}}{4^{m+1} k^{\prime 2} n^{2}} .
$$

As $\tau_{n}(x)$ is the approximating sum for $\varphi(x)$, the value of the integral would not be diminished if $\tau_{n}(x)$ were replaced by any other trigonometric sum of, order $n$ - in particular, by zero. That is, since $|\varphi(x)| \leqq \varepsilon$ and $0 \leqq \varphi(x) \leqq V$,

$$
\gamma_{n} \leqq 2 \pi V \varepsilon^{m}
$$

*It is immaterial whether the interval $(\alpha, \beta)$ is contained in $(0,2 \pi)$ or not, as the integral defining $\gamma_{n}$ can equally well be extended over any other interval of length $2 \pi$, because of the periodicity of the integrand. It may be assumed that $\beta-\alpha<2 \pi$, since otherwise the reasoning of the paper $\mathrm{A}$ would be directly applicable. 
Consequently, for $n \geqq n_{0}$,

$$
\frac{v \mu^{m}}{4^{m+1} k^{\prime 2} n^{2}} \leqq 2 \pi V \varepsilon^{m}, \quad \mu \leqq \sqrt[m]{\frac{8 k^{\prime 2} \pi V}{v}} \cdot 4 \varepsilon n^{2 / m}=C^{\prime} \varepsilon n^{2 / m},
$$

where $C^{\prime}$ depends on $m, V, v$, and $\beta-\alpha$, but not on $n$ or on $\varphi(x)$.

This is on the hypothesis that $\mu \geqq 4 \varepsilon$. But, in the contrary case, $\mu<4 \varepsilon \leqq 4 \varepsilon n^{2 / m}$, since it is understood that $n \geqq 1$, and if $C^{\prime \prime}$ stands for the larger of the numbers $4, C^{\prime}$, then

$$
\mu \leqq C^{\prime \prime} \varepsilon n^{2 / m}
$$

without exception, as soon as $n \geqq n_{0}$.

It will be recalled that $\mu$ is the maximum of $\left|\tau_{n}(x)\right|$ for $\alpha \leqq x \leqq \beta$, and that $|\varphi(x)| \leqq \varepsilon$ for all values of $x$. Hence

$$
\left|\varphi(x)-\tau_{n}(x)\right| \leqq \varepsilon+C^{\prime \prime} \varepsilon n^{2 / m} \leqq \varepsilon n^{2 / m}+C^{\prime \prime} \varepsilon n^{2 / m},
$$

or, if $C^{\prime \prime}+1=C$,

$$
\left|\varphi(x)-\tau_{n}(x)\right| \leqq C \varepsilon n^{2 / m}
$$

for $\alpha \leqq x \leqq \beta$ and for $n \geqq n_{0}$, the multiplier $C$, like $C^{\prime}$ and $C^{\prime \prime}$, being independent of $x, n$ and $\varphi$.

To apply this result, let $\varphi(x)$ be connected with the given function $f(x)$ by an identity of the form (4). Let such a function $\varphi$ be constructed for each value of $n \geqq 1$, the respective $\varphi$ 's being represented by the notation $\varphi_{n}(x)$, and the least upper bound of $\left|\varphi_{n}(x)\right|$ by $\varepsilon_{n}$. Let the approximating sum $\tau_{n}(x)$, of corresponding order $n$, be formed for each $\varphi_{n}$. As $\varphi_{n}(x)-\tau_{n}(x)$ is identical with $f(x)-T_{n}(x)$ for each value of $n$, where $T_{n}(x)$ is the approximating sum of the $n$th order for $f(x)$, it is seen that*

$$
\left|f(x)-T_{n}(x)\right| \leqq C n^{2 / m} \varepsilon_{n}
$$

for $\alpha \leqq x \leqq \beta$ and for $n \geqq n_{0}$.

* The reader may be assailed at first by a suspicion that this argument plays fast and loose with the subscript $n$. The fact is, however, that for independent $n$ and $\nu$

$$
\left|\varphi_{n}(x)-\tau_{\nu}(x)\right| \leqq C \nu^{2 / m} \varepsilon_{n}
$$

when $\nu \geqq n_{0}$, if $\tau_{\nu}(x)$ is the approximating sum of order $\nu$ for the function $\varphi_{n}(x)$; and then use is made of this relation, in the case of each $n \geqq n_{0}$, only for the single value $\nu=n$. The essential point is of course the fact that $C$ is independent of $\varphi$. 
If $f(x)$ is continuous, as must be assumed from now on, it follows from Weierstrass's theorem that the trigonometric sums $t_{n}(x)$ which define the remainders $\varphi_{n}$ can be chosen so that $\lim _{n=\infty} \varepsilon_{n}=0$. The rapidity with which $\varepsilon_{n}$ can be made to approach zero depends on further properties of $f(x)$, in a manner specified by various theorems on the approximate representation of continuous functions by trigonometric sums.* The significance of the relation (9) for the convergence of $T_{n}(x)$ can be expressed by the general statement

If the sums $t_{n}(x)$ can be chosen for all values of $n \geqq 1$ in such a way that

$$
\lim _{n=\infty} n^{2 / m} \varepsilon_{n}=0
$$

che approximating sum $T_{n}(x)$ will converge uniformly to the value of $f(x)$, as $n$ becomes infinite, throughout the interval $\alpha \leqq x \leqq \beta$; that is, throughout any interval in which (except possibly for a point set of measure zero) $\boldsymbol{o}(x)$ has a positive lower bound.

If $m=2$, for example, it is sufficient that $f(x)$ have a continuous derivativet for all values of $x$; if $m>2$, it is sufficient that $f(x)$ satisfy a Lipschitz condition, and sufficient, more generally, $\ddagger$ that $\lim _{\delta=0} \omega(\delta) / \delta^{2 / m}=0$, where $\omega(\delta)$ is the modulus of continuity of $f(x)$, the maximum of $\left|f\left(x^{\prime}\right)-f\left(x^{\prime \prime}\right)\right|$ for $\left|x^{\prime}-x^{\prime \prime}\right| \leqq \delta$.

In case the weight-function $\varrho(x)$ is continuous, the above results provide for the convergence of $T_{n}(x)$-apart from the question of uniform convergenceat all points where $\varrho \neq 0$, if $f(x)$ satisfies suitable conditions.

Although it is perhaps not worth while to use much space here in an attempt to lighten the restrictions on $f(x)$, it may be of some interest to point out one method of reasoning further from the stage that has been reached. $\S$ Let $\alpha$ and $\beta$ have the same significance as before, and let $\alpha_{1}$ and $\beta_{1}$ be two numbers such that $\alpha<\alpha_{1}<\beta_{1}<\beta$. For $\alpha_{1} \leqq x \leqq \beta_{1}$, the relation (6) may be replaced by the simpler statement that

$$
\left|\tau_{n}^{\prime}(x)\right| \leqq k_{1}^{\prime} n \mu
$$

* See e. g., D. Jackson, On approximation by trigonometric sums and polynomials, these Transactions, vol. 13 (1912), pp. 491-515; On the approximate representation of an indefinite integral, etc. (to be cited by the letter D), the same Transactions, vol. 14 (1913), pp. 343-364.

+ Cf. the paper D, Theorem IV, p. 351 .

+ Cf. $D$, p. 350. For the form of the result, cf. the papers $A$ and $C$.

$\S$ Cf. the paper $C, \S 6$. The method is carried further here than in the passage cited. 
where $k_{1}^{\prime}$ depends on $\alpha, \beta, \alpha_{1}$, and $\beta_{1}$, but not on anything else. Because of (8), this means that

$$
\left|\tau_{n}^{\prime}(x)\right| \leqq C_{1}^{\prime} \varepsilon n^{1+(2 / m)}
$$

for $n \geqq n_{0}, C_{1}^{\prime}$ being independent of $x, n$, and $\varphi$. Let $\mu_{1}$ be the maximum of $\left|\tau_{n}(x)\right|$ for $\alpha_{1} \leqq x \leqq \beta_{1}$, and let $x_{1}$ be a point in $\left(\alpha_{1}, \beta_{1}\right)$ such that $\left|\tau_{n}\left(x_{1}\right)\right|=\mu_{1}$. Let

$$
h_{1}=\frac{\mu_{1}}{2 C_{1}^{\prime} \varepsilon n^{1+(2 / m)}}
$$

it is clear that $C_{1}^{\prime} \neq 0$. Since $\mu_{1} \leqq \mu \leqq C^{\prime \prime} \varepsilon n^{2 / m}$, it follows that $h_{1} \leqq C^{\prime \prime} /\left(2 C^{\prime \prime} n\right)$, and there is a number $n_{1} \geqq n_{0}$, independent of $x, n$, and $\varphi$, such that $h_{1}<\frac{1}{2}\left(\beta_{1}-\alpha_{1}\right)$ for $n \geqq n_{1}$. When the last condition is satisfied, at least one of the intervals $\left(x_{1}-h_{1}, x_{1}\right),\left(x_{1}, x_{1}+h_{1}\right)$ will be contained in $\left(\alpha_{1}, \beta_{1}\right)$. Throughout one of these intervals, therefore,

$$
\left|\tau_{n}(x)-\tau_{n}\left(x_{1}\right)\right| \leqq \frac{\mu_{1}}{2}, \quad\left|\tau_{n}(x)\right| \geqq \frac{\mu_{1}}{2}
$$

Suppose temporarily that $\varepsilon \leqq \mu_{1} / 4 ;$ as $|\varphi(x)| \leqq \varepsilon$,

$$
\left|\varphi(x)-\tau_{n}(x)\right| \geqq \frac{\mu_{1}}{4}
$$

Since $\rho(x) \geqq v$ throughout the interval, except possibly for a set of measure zero,

$$
\gamma_{n} \geqq h_{1} v\left(\frac{\mu_{1}}{4}\right)^{m}=\frac{v \mu_{1}^{m+1}}{2 \cdot 4^{m} C_{1}^{\prime} \varepsilon n^{1+(2 / m)}}
$$

But, as before,

$$
\gamma_{n} \leqq 2 \pi V \varepsilon^{m}
$$

Hence

$$
\mu_{1} \leqq \sqrt[m+1]{\frac{C_{1}^{\prime} \pi V}{v}} \cdot 4 \varepsilon n^{\frac{m+2}{m(m+1)}}
$$

Under the hypothesis that $\varepsilon \leqq \mu_{1} / 4$, therefore, and also, as is recognized a posteriori, under the contrary hypothesis,

$$
\mu_{1} \leqq C_{1}^{\prime \prime} \varepsilon n^{\frac{m+2}{m(m+1)}}
$$


for $n \geqq n_{1}$, the factor $C_{1}^{\prime \prime}$ being independent of $x$, $n$, and $\varphi$.

This in itself would lead to an improvement in the convergence theorem, as far as the interval $\left(\alpha_{1}, \beta_{1}\right)$ is concerned. It is possible, however, to apply the same sort of reasoning again, in an interval $\left(\alpha_{2}, \beta_{2}\right)$, where $\alpha_{1}<\alpha_{2}<\beta_{2}<\beta_{1}$. More generally, let an infinite succession of such intervals be defined, each interior to the preceding; let $\mu_{i}$ be the maximum of $\left|\tau_{n}(x)\right|$ for $\alpha_{i} \leqq x \leqq \beta_{i}$, and suppose it has been shown that $\mu_{i-1} \leqq C_{i-1}^{\prime \prime} \varepsilon n^{\sigma}$ for $n \geqq n_{i-1}$, where $\sigma$ is some positive number depending only on $m$ and $i$, and $n_{i-1}$ is independent of $x, n$, and $\varphi$. By a series of steps analogous to those taken above,

$$
\left|\tau_{n}^{\prime}(x)\right| \leqq C_{i}^{\prime} \varepsilon n^{1+\sigma}
$$

for $\alpha_{i} \leqq x \leqq \beta_{i}$, with $C_{i}^{\prime}$ independent of $x, n$, and $\varphi$; for $n \geqq n_{i}$, a number independent of $x, n$, and $\varphi$, and for $\varepsilon \leqq \mu_{i} / 4$,

$$
\left|\varphi(x)-\tau_{n}(x)\right| \geqq \frac{\mu_{i}}{4}
$$

throughout an interval of length

$$
h_{i}=\frac{\mu_{i}}{2 C_{i}^{\prime} \varepsilon n^{1+\sigma}}
$$

wholly contained in $\left(\alpha_{i}, \beta_{i}\right)$; and so

$$
\begin{gathered}
\gamma_{n} \geqq h_{i} v\left(\frac{\mu_{i}}{4}\right)^{m}=\frac{v \mu_{i}^{m+1}}{2 \cdot 4^{m} C_{i}^{\prime} \varepsilon n^{1+\sigma}}, \\
\mu_{i} \leqq C_{i}^{\prime \prime} \varepsilon n^{\frac{1+\sigma}{m+1}}
\end{gathered}
$$

with $C_{i}^{\prime \prime}$ independent of $x, n$, and $\varphi$, the last relation holding whether the hypothesis $\varepsilon \leqq \mu_{i} / 4$ is verified or not.

Let $\sigma_{0}=2 / m$, and $\sigma_{i}=\left(1+\sigma_{i-1}\right) /(m+1), i=1,2, \cdots$. Then $\mu_{i} \leqq C_{i}^{\prime \prime} \varepsilon n^{\sigma_{i}}$ for each value of $i$, provided that $n>n_{i}$. It may be verified by induction that

$$
\sigma_{i}=\frac{1}{m}+\frac{1}{m(m+1)^{i}}
$$

the second term on the right approaching zero as $i$ becomes infinite. Let $\eta$ be an arbitrary positive quantity, and let $i$ be chosen so that $1 /\left[m(m+1)^{i}\right]<\eta$. 
Let $\alpha_{0}$ and $\beta_{0}$ be two arbitrary numbers, subject to the inequalities $\alpha<\alpha_{0}$ $<\beta_{0}<\beta$. The numbers $\alpha_{1}, \ldots, \alpha_{i}, \beta_{1}, \ldots, \beta_{i}$ may be chosen so that $\alpha_{i}=\alpha_{0}, \beta_{i}=\beta_{0}$. With the notation $\mu_{i}=\mu_{0}, C_{i}^{\prime \prime}=C_{0}^{\prime \prime}, n_{i}=n_{0}^{\prime}$, it is seen that

$$
\mu_{0} \leqq C_{0}^{\prime \prime} \varepsilon n^{(1 / m)+\eta}
$$

for $n \geqq n_{0}^{\prime}$, where $\mu_{0}$ now is the maximum of $\left|\boldsymbol{\tau}_{n}(x)\right|$ for $\boldsymbol{\alpha}_{0} \leqq x \leqq \boldsymbol{\beta}_{0}$. By an argument which need not be repeated in detail, it is recognized that $T_{n}(x)$ converges uniformly to $f(x)$ throughout an arbitrary closed interval interior to $(\alpha, \beta)$, if there exists a number $\eta>0$ such that

$$
\lim _{\delta=0} \omega(\delta) / \delta^{(1 / m)+\eta}=0
$$

$\omega(\delta)$ being once more the modulus of continuity of $f(x)$. If $f(x)$ satisfies a Lipschitz condition, this requirement will be fulfilled for any value of $m>1$.

As before, the results are applicable, apart from the question of us. formity, at any point where $\varrho \neq 0$, if $\varrho$ is continuous. The probiem of $\mathrm{c}_{2}$ vergence at a point where $\varrho$ vanishes will not be treated at length, but will be touched upon just to the extent of showing that it is not wholly beyond the reach of the methods that have been employed.

Let it be supposed that an interval $(\boldsymbol{\alpha}, \boldsymbol{\beta})$ and positive numbers $w$ and $s$ exist so that*

$$
\varrho(x) \geqq w(x-\alpha)^{s}
$$

for $\alpha \leqq x \leqq \beta$. Further hypotheses with regard to $\varrho$ are merely that it is everywhere bounded and measurable, and of period $2 \pi$. With the sam. notation as before, and by the same reasoning, there is an interval of length at least $h=1 /\left(4 k^{\prime 2} n^{2}\right)$, contained in $(\alpha, \beta)$ for $n \geqq n_{0}$, throughout which $\left|\varphi(x)-\tau_{n}(x)\right| \geqq \mu / 4$, if $\mu \geqq 4 \varepsilon$. Let the left-hand end of this interval, $x_{0}$ or $x_{0}-h$, according to circumstances, be denoted by $\alpha^{\prime}$.

* It would come to the same thing if it were assumed that $\rho(x) \geqq v(\beta-x)^{*}$, or that $\alpha$ is interior to an interval throughout which $\rho(x) \geqq w|x-\alpha|^{*}$. The essential point is that, for approach from one side at least, $\rho$ has a root of order not higher than $s$. The most interesting special values of $s$ are presumably $s=2$, the lowest value consistent with the existence of a derivative for $\rho$ at the point in question, and $s=1$. 
This $\alpha^{\prime}$ may or may not coincide with $\alpha$, but in any case $0 \leqq x-\alpha^{\prime} \leqq x-\alpha$ for $\alpha^{\prime} \leqq x \leqq \beta$, and so

$$
\rho(x) \geqq w(x-\alpha)^{s} \geqq w\left(x-\alpha^{\prime}\right)^{s}
$$

for $\boldsymbol{\alpha}^{\prime} \leqq x \leqq \boldsymbol{\alpha}^{\prime}+h$. Consequently

$$
\begin{array}{r}
\gamma_{n}=\int_{0}^{2 \pi} \rho(x)\left|\varphi(x)-\tau_{n}(x)\right|^{m} d x \geqq w\left(\frac{\mu}{4}\right)^{m} \int_{\alpha^{\prime}}^{\alpha^{\prime}+h}\left(x-\alpha^{\prime}\right)^{s} d x \\
=w\left(\frac{\mu}{4}\right)^{m} \cdot \frac{h^{s+1}}{s+1}=\frac{w \mu^{m}}{4^{m+s+1}(s+1)\left(k^{\prime} n\right)^{2 s+2}} .
\end{array}
$$

On the other hand, as always, $\gamma_{n} \leqq 2 \pi V \varepsilon^{m}$. So

$$
\mu \leqq \bar{C} \varepsilon n^{(2 s+2) / m}
$$

for $n \geqq n_{0}$, the values of $n_{0}$ and $\bar{C}$ being independent of $x, n$, and $\varphi$, and the conclusion valid even if the hypothesis that $\mu \geqq 4 \varepsilon$ is dropped. If $m=2$ and $s=1, T_{n}(x)$ will converge to $f(x)$ for $x=\alpha$ (and uniformly for $\alpha \leqq x \leqq \beta)$ if $f(x)$ has everywhere a continuous second derivative,* while the existence of a continuous third derivative will be sufficient if $m=s=2$.

* For the requisite preliminary theorem on the approximate representation of $f(x)$ bv means of trigonometric sums, cf. once more the paper D, Theorem IV, p. 351.

The University of Minnesota,

Minneapolis, Minn. 